Electronic Journal of Research

\title{
Promoción de la prosocialidad y la justicia social en educación primaria: una experiencia preliminar
}

\section{Miguel Ángel Albalá Genol ${ }^{1}$, Jesús Guerra Gamero ${ }^{2}$}

\author{
${ }^{1}$ Universidad Internacional de Valencia, Valencia. \\ ${ }^{2}$ Departamento de Psicología Evolutiva y de la Educación, \\ Universidad Autónoma de Madrid.
}

\section{España}

Correspondencia: Miguel Ángel Albalá Genol, Universidad Internacional de Valencia.

E-mail: miguelangel.albala@campusviu.es

(C) Universidad de Almería and Ilustre Colegio Oficial de la Psicología de Andalucía Oriental (Spain) 


\section{Resumen}

Introducción. Desde hace décadas se ha estudiado el concepto de Justicia Social en la educación y las posibles implicaciones que tiene como herramienta para promover sociedades más prosociales. A partir del modelo multidimensional de Justicia Social de Nancy Fraser, se diseñó un programa para promover la prosocialidad en estudiantes de $6^{\circ}$ curso de educación primaria. Los principales objetivos de estudio fueron conocer las representaciones de Justicia Social que tenía el alumnado y promover la prosocialidad mediante la implementación preliminar de un programa socioeducativo diseñado.

Método. La metodología se basó en talleres eminentemente participativos e inspirados en el aprendizaje por cooperación. Después de una evaluación inicial de las representaciones de la Justicia Social del alumnado, se realizó una implementación preliminar de un programa socioeducativo diseñado en 84 estudiantes de diversos centros educativos de Madrid (España). Dicho programa se estructuró en tres módulos principales: 1) Descubriendo la Justicia Social; 2) Habilidades para descubrir y solucionar injusticias sociales; y 3) Creando un marco común de Justicia Social para convivir prosocialmente. Al finalizar el programa fueron evaluados cuantitativamente los cambios producidos en las representaciones de Justicia Social del alumnado.

Resultados. Los resultados mostraron mejoras en las representaciones de Justicia Social evaluadas al inicio del programa preliminar, así como, en la capacidad de identificación de injusticias sociales derivadas de los diversos trilemas planteados. Además, se evidenciaron mejoras significativas en la mayoría de los trilemas evaluados, mostrando el alumnado una mayor prosocialidad en el abordaje de estos. Así, el programa socioeducativo se mostró eficaz independientemente del sexo y el tipo de centro educativo al que pertenecía el alumnado participante.

Discusión y conclusiones. En base a esta experiencia preliminar aplicada se discuten los alcances de la metodología socioeducativa de cara a promover la prosocialidad mediante los principios basados en la Educación para la Justicia Social. Se concluye que programas de este tipo pueden promover una convivencia más prosocial del alumnado fuera y dentro de las aulas, fomentando en estos una mayor comprensión de las injusticias vividas por otras personas. Por último, exponen algunas limitaciones detectadas y posibles mejoras para la implementación definitiva del programa en el futuro.

Palabras Clave: Prosocialidad, Ciudadanía, Educación, Intervención Socioeducativa. 


\section{Abstract}

Introduction. For decades the Social Justice concept in education has been studied alongside and the possible implications of its use as a tool to promote more prosocial societies. Based on Nancy Fraser's multidimensional Social Justice Model, a program was designed to promote prosociality in 6th grade primary school students. The main objectives of this research were to become acquainted with the student's representations of Social Justice and to promote prosociality through a preliminary implementation of a designed educational program.

Method. The methodology was based on eminently participative workshops inspired by cooperative learning. After an initial evaluation of the students' representations of Social Justice, a preliminary implementation of a socio-educational program was carried out with 84 students from various educational schools in Madrid (Spain). This program was structured in three main modules: 1) Discovering Social Justice; 2) Skills to discover and solve social injustices; and 3) Creating a common framework of Social Justice to coexist prosocially. At the end of the program, the changes in the Social Justice representations of the students were quantitatively evaluated.

Results. The results showed improvements in the Social Justice representations that were evaluated at the beginning of the preliminary program, as well as in the ability to identify social injustices derived from the various trilemmas analyzed. Furthermore, significant improvements were evidenced in most of the trilemmas evaluated, showing that the students demonstrated a greater prosociality in their approach. Thus, the socio-educational program was effective regardless of gender and type of school to which the participating students belonged.

Discussion and Conclusion. Based on this applied preliminary experience, the scope of the socio-educational methodology is discussed in order to promote prosociality through the principles based on Education for Social Justice. We concluded these kinds of programs are capable of promoting a more prosocial coexistence of the students inside and out of the classroom, promoting a greater understanding of the injustices lived by other people. Finally, some detected limitations and possible improvements for the definitive implementation of the program in the future are also discussed.

Keywords: Prosociality, Citizenship, Education, Socioeducational Intervention. 


\section{Introducción}

Desde numerosas disciplinas, se ha estudiado y desarrollado el concepto de Justicia Social, así como sus posibles implicaciones como herramienta para avanzar hacia sociedades más prosociales y equitativas. Además, dichos estudios han realizado aportes al desarrollo de campos tales como la filosofía, la psicología, la sociología o la política. Sin embargo, todo este poderoso arsenal académico a veces ha tenido menos relevancia a la hora de ser implementado en el campo de la educación aplicada. No obstante, si se han desarrollado programas de intervención que a través del papel de las habilidades sociales y emocionales han buscado el ajuste social del alumnado y el profesorado, así como el fomento de la empatía y la prosocialidad (Spinrad y Gal, 2018). En este sentido, en el escenario educativo actual marcado por el incremento de grupos minoritarios y diversos en las aulas, el desarrollo de propuestas inspiradas en principios de inclusión, prosocialidad, multiculturalidad y Justicia Social cobran cada vez más relevancia (Jiménez, Lalueza y Fardella, 2017). Así, se hace explícita la necesidad de incluir en la práctica todas estas contribuciones teóricas de cara a poder continuar avanzando en este campo. En este sentido, partiendo de un enfoque de Educación para la Justicia Social (Murillo y Hernández-Castilla, 2014), se persigue que esta sea la herramienta fundamental a la hora de luchar contra la desigualdad, la exclusión, la discriminación y/o la marginación social (Jacott y Maldonado, 2012).

Actualmente, son numerosas las injusticias que pueden observarse tanto en nuestro contexto más inmediato (a nivel local, autonómico y estatal), como en contextos más lejanos (a nivel europeo e internacional). Por un lado, dichas injusticias están conformadas por formas de discriminación y marginalización que muchas veces vienen afectando a la sociedad desde siglos atrás (racismo, machismo, homofobia, clasismo, entre otras). Por otro lado, continuamente aparecen nuevos tipos de injusticias relacionadas con fenómenos más actuales, como: la aporofobia (Martínez, 2002), que trata de excluir a los más pobres de la sociedad; y más centrado en el campo educativo, aparece el acoso escolar o bullying (Olweus, 1994; Mateu-Martínez, Piqueras, Rivera-Riquelme, Espada y Orgilés, 2014) y más recientemente el ciberbullying (Di Lorenzo, 2012). Reducir y prevenir los comportamientos y actitudes en relación a las anteriores injusticias, deben ser objetivos a tener en cuenta si se pretende que las generaciones futuras no continúen marginalizando a una parte importante de la sociedad. Además, existe otra poderosa razón que pone el foco en la necesidad de trabajar sobre estos principios, esta es, la mejora de la convivencia dentro de los centros educativos. La revisión 
de algunos estudios realizados indica que la existencia del maltrato entre iguales en España es un fenómeno que se produce en todas las clases sociales y tipos de centros educativos (Garaigordobil y Oñederra, 2008), considerándose como una realidad social que mantiene un patrón de comportamiento estable (Monjas, Martín-Antón, García-Bacete y Sanchiz, 2014).

Según Nocito Muñoz (2017), la mayoría de las intervenciones para evitar el acoso escolar se centran en la etapa adolescente, sin embargo, a nivel europeo los datos indican que las víctimas más vulnerables son los escolares entre 8 y 11 años (Rajmil, Alonso, Analitis, Detmar, Erhart, Klein, Ravens-Sieberer, Herdman y Berra, 2009), coincidiendo con el último ciclo de primaria. En esta misma línea, existen estudios que evidencian que los conflictos y problemas de convivencia entre el alumnado no son hechos exclusivos de la educación secundaria, sino que su incidencia comienza en edades más tempranas (Sánchez, 2009). Además, autores como Eisenbraun (2007) apuntan la necesidad de afrontar estas situaciones a través de programas de prevención y de promoción. Desde su contexto más cercano, el alumnado de educación primaria puede comenzar a sentar las bases de la convivencia y respeto hacia los demás.

El papel de la educación en la promoción de la igualdad y la Justicia Social es una de las principales preocupaciones de los políticos que desempeñan un papel central a la hora de decidir qué se enseña en nuestras escuelas (Smith, 2018). Sin embargo, la educación es un proyecto político integrado dentro de una red de instituciones sociales que muchas veces reproducen desigualdades (Sensoy y DiAngelo, 2017). En las últimas décadas, en el campo educativo se han producido dos grandes hitos en torno a un concepto tan tremendamente subjetivo como es el de Justicia Social. Por un lado, cada vez son más las escuelas e instituciones educativas, que se han puesto de acuerdo en la necesidad de introducir dicho concepto en la educación (García-Lastra, 2013). Y, por otro lado, se ha creado una base sólida sobre la que trabajar la Justicia Social mediante una perspectiva tridimensional (Fraser, 2008) basada en tres dimensiones (Redistribución, Reconocimiento y Representación) para la búsqueda de la inclusión educativa (Simón, Barrios, Gutiérrez, Muñoz, 2019). En base a esta concepción, en primer lugar, aparece el concepto de Redistribución (Rawls, 1971; Sen 2012), refiriéndose a la necesidad de una mejor distribución de los bienes materiales y recursos culturales. En segundo lugar, en referencia al Reconocimiento (Fraser, 1997; Irvine, 2003), este se centra en la necesidad de ausencia de dominancia social y de respeto y valoración de todos los grupos sociales y personas. En tercer lugar, aparece la dimensión Participación (Bell, 1997; Lee y 
Hipólito-Delgado, 2007) se refiere a la necesidad de participación y representación, para que todas las personas y grupos tengan capacidad de decisión en aspectos que afectan a sus propias vidas y a la sociedad en general. Así, ni la redistribución de la riqueza, ni el reconocimiento a la diversidad tienen sentido sin una representación real, tratándose de tres factores necesarios en una sociedad justa y no siendo suficiente para ello ninguna de las tres dimensiones de forma aislada (Fraser, 2008).

Este enfoque tridimensional posiblemente esté ayudando a disminuir algunas de las dificultades que tradicionalmente habían aparecido para trabajar este concepto. Así, y en relación a la justicia social, una de las dificultades recae en que no tiene un significado único, siendo altamente político (Murillo y Hernández-Castilla, 2011). Partiendo de esta perspectiva, algunos estudios ya han mostrado que existen diferencias en la representación de cada una de las tres dimensiones de Justicia Social que tiene el alumnado y profesorado. Así, según Juanes, García, Maldonado y Jacott (2016) se concluye que en sexto curso de primaria las alumnas tienen una representación más prosocial que los alumnos, tanto en las dimensiones redistribución, como en reconocimiento. En cuanto a la dimensión representación/participación, en este mismo curso, son los alumnos los que cuentan con representaciones más prosociales, al igual que el alumnado que procede de un contexto socioeconómico menos favorecido (Juanes, 2018). De la misma manera, en niveles educativos superiores (secundaria y bachillerato) también se encontraron diferencias en función del sexo del alumnado (Sainz y Jacott, 2020) siendo las mujeres quienes mostraban representaciones más prosociales.

En el análisis psicoeducativo relacionado con la conducta prosocial se hace necesario un abordaje complejo, teniendo en cuenta la multitud de factores interrelacionados de los que depende (Garaigordobil, 2003). En los últimos años, las actitudes y conductas prosociales han sido objeto de estudio para la Psicología y la Educación, teniendo en cuenta no solo el contexto escolar sino también el familiar, el cultural, y otros factores vinculados a la personalidad o la biología. Actualmente, existe evidencia acerca de la mediación que tienen diversos factores socioculturales (normas socioculturales, valores y estándares de una sociedad, entre otros), que los/as más jóvenes interiorizan en el proceso de socialización en relación a la implementación de conductas prosociales con respecto a sus iguales (Garaigordobil, 2014). Así, en ciertas culturas orientales son más valoradas por la sociedad las conductas prosociales, así como el hecho de que entre las personas más jóvenes exista una interacción cooperativa. Actualmente, es aceptado que cada cultura promueve diferentes valores sociomorales, teniendo ello 
un papel clave tanto en la frecuencia de la conducta prosocial de diferentes colectivos, como en la justificación y el razonamiento ofrecidos en la argumentación de ayudar o no a otras personas (Duque, 2017). En este sentido, ante sociedades occidentales y sistemas educativos en los que frecuentemente se exige para el éxito la competitividad individualista, la cultura educativa (escolar y familiar) se sitúa como un elemento clave en la promoción de la prosocialidad. En este proceso la familia desempeña un rol importante, pero la adquisición de las conductas prosociales se da de forma relevante en el contexto escolar, que según Garaigordobil (2014): "tiene por meta prioritaria el desarrollo moral enfatizando en la enseñanza de la cooperación con los demás mediante la utilización de prácticas y programas eductivos que promueven la prosocialidad mediante modelos de conducta" (p. 153).

En esta línea, mediante su trabajo y su liderazgo, los profesionales de la educación son modelos de referencia para el alumnado y, por tanto, con capacidad para cambiar la forma de percibir la realidad (Jornet-Meliá, Sancho-Álvarez y Bakieva, 2015). Según McAuliffe, Hubbard y Romano (2009), las cogniciones del profesor (imagen positiva del alumnado versus imagen de estos como personas agresivas) y sus conductas correctivas o negativas hacia el alumnado, median la conducta prosocial y agresiva. Así, puede ser clave que ya desde la educación primaria, el alumnado conozca información relacionada con los niveles de justicia e injusticia social con los que convive en términos generales (a nivel internacional) y en términos particulares (a nivel nacional, de comunidad autónoma y local), esto es, Educación en Justicia Social (Murillo y Hernández Castilla, 2011). Para poder establecer estrategias justas de participación, un buen paso previo puede ser comprender las injusticias sociales que rodean la realidad en la que vivimos y cómo afrontarlas. Por ejemplo, se ha encontrado una relación clara entre las ideas de los estudiantes sobre la Justicia Social y su implicación y compromiso con las escuelas (Miller, Williamson, George, King, Charest, Bieler y Bolf Beliveau, 2011). En esta línea, también se evidencia la importancia del factor familiar y las limitaciones de las políticas educativas participativas, que reflejan carencias en el análisis de las estrategias familiares que están detrás de la reproducción de las desigualdades sociales (Morgade, Poveda, González-Patiño, 2014). Así, algunos programas han utilizado elementos de juego serio para introducir el tema complejo de las conexiones económicas globales (relacionadas con la justicia económica o redistribución) de una manera lúdica (Wartenweiler, 2018). También, diversos programas de intervención psicoeducativos (Cerchiaro-Ceballos, Barras-Rodríguez y Vargas-Romero, 2019; Lozano y Lechuga, 2019), han encontrado un mayor desarrollo proso- 
cial en el alumnado que se implicaba en los mismos, alcanzando niveles de razonamiento prosocial de una mayor complejidad.

En base a ello, el presente estudio trata de incorporar la Educación para la Justicia Social mediante el diseño de un programa socioeducativo, basado en metodología cooperativa orientada hacia la educación inclusiva (Cabrera y Davyt, 2015) que es considerada como una investigación para la transformación de la intervención educativa y social. Este enfoque metodológico es analítico con la realidad en la que se desarrolla, partiendo frecuentemente hacia la promoción del pensamiento crítico. Desde esta perspectiva, una herramienta relevante para el alumnado puede ser introducir la participación como una estrategia de acción de cara a resolver justamente los posibles problemas cotidianos, así como para llevar a cabo a mediolargo plazo una transformación hacia sociedades más justas y democráticas. Esta participación, debe ser transmitida como el mecanismo básico de adquisición de dominio y de control que se asocia con la posibilidad de influir en el entorno (Rappaport, 1977). Así, la participación proporciona oportunidades para aprender, perfeccionar y poner en práctica habilidades relacionadas con la toma de decisiones y la solución de problemas (Zimmerman, 2000).

El principal objetivo del presente estudio fue la implementación preliminar del programa socioeducativo "EJUSO: Educando en Justicia Social”, de cara a conocer los cambios en la prosocialidad del alumnado de $6^{\circ}$ curso de Educación Primaria. En este sentido, el estudio pretende analizar, antes y después del programa de intervención, la capacidad de resolución justa del alumnado en torno a trilemas que requieren de actitudes prosociales. Dicho programa tuvo dos objetivos principales: 1) analizar la representación, conocimientos y habilidades iniciales del alumnado en relación a la Justicia Social; 2) conocer los cambios en el pensamiento prosocial y las representaciones de Justicia Social del alumnado tras la intervención. Las hipótesis de estudio planteadas fueron tres:

- Hipótesis 1: "El programa socioeducativo será eficaz para mejorar las tres dimensiones desde las que se promociona la justicia social”.

- Hipótesis 2: “Tras la intervención preliminar el alumnado mostrará representaciones más prosociales de la justicia social, independientemente de su sexo".

- Hipótesis 3: “Tras la intervención preliminar el alumnado mostrará representaciones más prosociales de la justicia social, independientemente del centro educativo al que pertenece". 


\section{Método}

\section{Participantes}

En este estudio preliminar participaron 84 estudiantes de $6^{\circ}$ curso de primaria, distribuidos por sexo en 38 alumnas y 46 alumnos pertenecientes a dos escuelas públicas (30 participantes del CEIP Guindalera y 20 participantes del CEIP San Ildefonso) y una privada (34 participantes de la Escuela Privada Ideo) de Madrid. Estos centros no contaban con ningún tipo de programación educativa relacionada con temáticas afines a la educación prosocial y para la Justicia Social. Para la aplicación preliminar se contó con centros educativos tanto privados como públicos, de cara a conformar grupos de alumnado con características heterogéneas. Los tres centros educativos aceptaron participar voluntariamente en el programa, tras ser informados previamente del contenido del mismo y previa firma del documento de consentimiento informado por parte de los padres o tutores legales en cada caso.

\section{Instrumentos}

Al inicio y finalización del programa preliminar implementado se empleó el "Cuestionario de Representación de Justicia Social en estudiantes de primaria-CRJS” (Juanes, 2018), diseñado y validado anteriormente para explorar la representación de Justicia Social, entendida como: "La capacidad analítica y de resolución justa de trilemas morales que contienen situaciones injustas relacionadas con las dimensiones redistribución, reconocimiento y representación". Dicho cuestionario, contiene 21 trilemas sobre las tres dimensiones de Justicia Social (redistribución, reconocimiento y representación), relacionados con el análisis y resolución prosocial de injusticias relacionadas con la escuela y contextos cotidianos para el alumnado. Este cuestionario fue objeto de validación en trabajos anteriores (Jacott, Maldonado Sainz, Juanes, García-Vélez y Seguro, 2014) llevando a cabo un proceso de validación por juicio de expertos, en el que se validó cada una de las tres alternativas de los dilemas en una escala Likert de justicia social mostrando un buen coeficiente de correlación intraclase $(C C I=$ 0.97), así como en relación a otros índices de validación $(C V R t=0.67, W$ de Kendall $=0.62$, Kappa del coeficiente de Cohen $=0.48$ ). Además, la escala mostró una fiabilidad adecuada en su validación, analizada mediante el indice alfa de Cronbach $(\alpha=.74)$. En cada trilema, primero, se describe brevemente un problema o situación relacionada con las dimensiones de Justicia Social y luego el alumnado de primaria puede elegir entre una de las tres posibles respuestas: Una que promueve la Justicia Social; una segunda respuesta que es neutral o menos promotora de la Justicia Social que la anterior; y otra que va contra la Justicia Social. Con 
este cuestionario es posible obtener un índice global de la representación de Justicia Social y un índice específico para cada una de las tres dimensiones. Estos índices están adaptados para tener un rango de 1 a 100. A modo de ejemplo se puede ver el siguiente trilema utilizado en el cuestionario, que se relaciona con la dimensión de redistribución. La opción que representa una solución más justa sería la A, luego la opción C representa una solución menos justa que la anterior, y por último la opción B representa la solución menos justa de todas:

"Pablo es un niño de $6^{\circ}$ primaria al que un grupo de su clase molesta y acosa continuamente. Santiago sabe lo que están haciendo sus amigos y amigas y quiere pararlo. ¿Qué es lo que debería hacer?"

A) Decirles que no pueden hacerle eso a Pablo y, si no le hacen caso, pedir ayuda a un adulto.

B) No intervenir en las disputas, para no perder a sus amigas y amigos.

C) Hablar con Pablo para ayudarle a evitar esas situaciones.

\section{Procedimiento}

Para el estudio se planteó un diseño de investigación cuantitativo cuasi-experimental, en el que se evaluaron los cambios en la prosocialidad del alumnado por medio de un planteamiento pretest-postest. No se pudo contar con grupos control en el presente estudio, dada la dificultad de acceso a los centros educativos que demandaban que todo el alumnado participara integramente en el programa. El tipo de muestreo que se realizó para el estudio fue no probabilístico incidental (Sampieri, Fernández y Baptista, 2014), pues la muestra de alumnado fue elegida en base a la accesibilidad permitida por los centros educativos. Al comienzo de la implementación preliminar del programa, se evaluó la representación inicial de Justicia Social del alumnado mediante el CRJS. El programa se encontraba formado por tres módulos principales, con sesiones compuestas por actividades y talleres que tenían una duración de 50 minutos, lo cual corresponde con el transcurso aproximado de una clase habitual dentro de cada centro. La extensión total de la implementación fue de ocho sesiones, distribuidas en ocho semanas consecutivas. Después de completar los tres módulos de actividades y talleres, fue evaluada nuevamente la representación de Justicia Social con la misma herramienta ya empleada al comienzo, de cara a analizar los posibles cambios y efectividad del programa.

En referencia a los contenidos que conforman el programa de intervención socioeducativa, este consta de tres módulos principales: 1) Aproximación al concepto de Justicia Social; 2) Descubriendo qué es y qué no es la Justicia Social; 3) Concluyendo un marco común 
de convivencia en Justicia Social. Para esta fase, se tuvieron especialmente en cuenta los factores facilitadores en el proceso de implementación (Murillo y Krichesky, 2012), buscando un equilibrio entre los ejes: Simplicidad-complejidad; Flexibilidad-rigidez; Fidelidad-adaptación. En lo relativo a los contenidos del programa socioeducativo, el primer módulo se centra en explorar el concepto de Justicia Social que posee el alumnado de sexto de primaria. A partir de este primer módulo, se introduce el modelo tridimensional de Justicia Social a través de dos actividades prácticas. La primera actividad consiste en el "Concurso de Justicia Social" centrado en buscar la cooperación entre los y las participantes como condición imprescindible para poder participar de manera efectiva. Al inicio, se formaban tres grupos de trabajo (cada uno de ellos encargado de asesorar de una dimensión de Justicia Social). Para ello, a cada grupo se le proporcionaba información y datos relacionados con las preguntas referentes a la dimensión que representan, y que los otros dos grupos tendrán que responder a lo largo del concurso. A modo de concurso, se le plantean a cada grupo una serie de preguntas relacionadas con alguna de las dos dimensiones de Justicia Social de las que no tienen información. De esta manera, siempre deben apoyarse para responder, con un tiempo limitado, en el grupo que si posee información relacionada con la dimensión sobre la que se pregunta. El equipo que colabora podrá aportar pistas en relación a la pregunta, de acuerdo a la información que posee. Aunque hay tres grupos de trabajo diferenciados por cada dimensión de Justicia Social, el objetivo es común: acertar entre todos los grupos el mayor número de respuestas.

En la segunda actividad de este primer módulo, el alumnado a través de juegos colaborativos, definen con sus propias palabras y utilizan conceptos tales como: Feminismo, racismo, sexismo, clasismo, igualdad, pobreza, integración, democracia, libertad, etc. A su vez, otra tarea de esta actividad es asociar de forma argumentada cada concepto trabajado con una o varias dimensiones de Justicia Social, así como, contrastar las definiciones dadas inicialmente con las mayoritariamente aceptadas en la actualidad.

En el segundo módulo del programa el propósito es identificar los tipos de injusticias sociales y el origen de las mismas, así como formas de participación individuales y colectivas de cara a poder revertirlas. Se desarrollan varias actividades prácticas basadas en role-play en las que deben implicar activamente la identificación y resolución de situaciones reales o hipotéticas que ocurren en su vida cotidiana. Con cada situación, se persigue que los y las participantes adquieran los diferentes puntos de vista que ocurren en situaciones de injusticia, fomentando especialmente tanto la identificación de injusticias y la empatía hacía quien las pa- 
dece, como diversas habilidades sociales y de resolución de las mismas mediante la participación. Finalmente, se construyen de forma consensuada posibles formas de resolución de las injusticias planteadas, en base a las tres dimensiones de Justicia Social planteadas.

En el tercer módulo del programa se evalúa si ha cambiado la representación de Justicia Social que tenían los y las participantes antes y después del mismo (evaluación pre-post de cada trilema mediante el CRJS). Se plantea un debate general en donde el alumnado transmite los conceptos principales que han aprendido durante las sesiones previas. En esta fase, se trata de fomentar que el alumnado participante exprese sus propias definiciones de los conceptos trabajados, así como la forma en la que plantean trasladarlos a su realidad, tanto dentro como fuera del aula. Para finalizar, todo ello se sintetiza mediante una actividad basada en la realización de un mural grupal, a través de la cual se incorpora una concepción de Justicia Social consensuada por todos y todas. Del mismo modo, este mural supone un compromiso por parte del grupo para mejorar y mantener un clima justo de convivencia en el aula. Al finalizar este módulo, se volvió a evaluar la representación de Justicia Social del alumnado, mediante el cuestionario también cumplimentado al inicio.

\section{Análisis de datos}

De cara al análisis de datos, se utilizó el programa SPSS versión 24 para Windows 10. En primer lugar, se obtuvieron los índices de representación de Justicia Social en cada una de las tres dimensiones evaluadas pre-post intervención mediante el Cuestionario de Representación de Justicia Social, calculando los estadísticos descriptivos $(M ; D T)$ para cada trilema. Luego, se empleó una Prueba T de Student para muestras relacionadas, de cara conocer los cambios pretest-postest en la capacidad de resolución prosocial en cada trilema planteado. Además, para conocer con mayor profundidad los cambios en la prosocialidad del alumnado, se analizaron las diferencias pretest-postest del alumnado específicamente en cada trilema. Por último, se aplicó una Prueba T de Student para muestras independientes, con el objetivo de analizar estadísticamente las posibles diferencias pretest-postest en función del sexo y del tipo de centro educativo. El nivel de significancia se estableció en $p<.05$.

\section{Resultados}

En primer lugar, los resultados muestran cambios pretest-postest en la prosocialidad del alumnado hacia cada una de las dimensiones de Justicia Social analizadas. En este sentido, 
se evidencia que tras la implementación del programa (post-test), se encuentran mejoras estadísticamente significativas en la resolución prosocial de trilemas relacionados con las dimensiones Reconocimiento y Participación. Como observa en la Tabla 1, en la evaluación postest el alumnado muestra puntuaciones medias superiores en todos los casos, con respecto a la evaluación pretest.

Tabla 1. Puntuaciones promedio pre-post intervención en el alumnado participante.

\begin{tabular}{|c|c|c|c|c|c|c|c|c|c|}
\hline & $\begin{array}{c}\text { Redistrib. } \\
\text { Pre }\end{array}$ & $\begin{array}{c}\text { Redistrib. } \\
\text { Post }\end{array}$ & Sig. & $\begin{array}{c}\text { Reconoc. } \\
\text { Pre }\end{array}$ & $\begin{array}{c}\text { Reconoc. } \\
\text { Post }\end{array}$ & Sig. & $\begin{array}{c}\text { Partic. } \\
\text { Pre } \\
\end{array}$ & $\begin{array}{c}\text { Partic. } \\
\text { Post }\end{array}$ & Sig. \\
\hline$M$ & 16.35 & 16.93 & $p=.34$ & 16.40 & 17.38 & $p=.037$ & 15.69 & 17.38 & $p<.001$ \\
\hline$D T$ & 2.18 & 2.05 & & 1.77 & 1.71 & & 2.19 & 1.89 & \\
\hline
\end{tabular}

Fuente: Elaboración propia a partir de los resultados de la investigación.

Específicamente en relación a las comparaciones intragrupo entre las fases pretestpostest, los resultados indican que el alumnado participante obtiene puntuaciones significativamente más elevadas en la fase postest en Reconocimiento $\left(t_{(84)}=3.65 ; p=.037\right)$ y Participación $\left(t_{(84)}=5.88 ; p<.001\right)$. Dichas puntuaciones muestran que no fueron significativas las mejoras en la dimensión Redistribución $\left(t_{(84)}=2.18 ; p=.34\right)$ en comparación con la fase pretest.

Posteriormente, se analizaron las diferencias pretest-postest específicamente en cada uno de los 21 trilemas sometidos a estudio. Como se muestra en la Tabla 2, se evidenciaron diferencias estadísticamente significativas en las puntuaciones medias pretest-postest en $12 \mathrm{de}$ los 21 trilemas analizados. Dichas diferencias se hallaron distribuidas de forma heterogenea en las tres dimensiones de Justicia Social abordadas, encontrando mayores cambios en la prosocialidad hacia la dimensión Participación, seguida de las dimensiones Reconocimiento y Redistribución. En primer lugar, con respecto a la dimensión Redistribución, se encontraron diferencias significativas pretest-postest $(p<.05)$ en los trilemas 14 y 19. En dichos trilemas se evalúa la prosocialidad hacia cuestiones relacionadas con las becas educativas y la desigualdad de recursos entre el alumnado. En segundo lugar, en relación a la dimensión Reconocimiento, se hallaron también diferencias significativas pretest-postest $(p<.05)$ en los trilemas 1 , 2, 16 y 18. Dichos trilemas se encuentran relacionados con la tolerancia hacia: la población migrante, el alumnado con movilidad reducida, y la diversidad sexual y de género entre las familias del alumnado. En tercer lugar, en la dimensión Participación, es en la que se encontraron diferencias significativas pretest-postest $(p<.05)$ en un mayor número de trilemas (tri- 
lemas 4, 8, 9, 10, 12 y 15). Estos trilemas estuvieron específicamente relacionados con la participación democrática, la participación del alumnado dentro y fuera del aula, y la participación del alumnado y profesorado hacia la resolución de situaciones de acoso en el contexto escolar.

Tabla 2. Puntuaciones medias y diferencias pre-post intervención en cada trilema evaluado.

\begin{tabular}{|c|c|c|c|c|c|c|}
\hline \multirow[t]{3}{*}{ Redistribución } & \multirow{2}{*}{\multicolumn{2}{|c|}{ Pre }} & \multirow{2}{*}{\multicolumn{2}{|c|}{ Post }} & \multirow[b]{3}{*}{$t(g l)$} & \multirow[b]{3}{*}{ Sig. } \\
\hline & & & & & & \\
\hline & $M$ & $D T$ & $M$ & $D T$ & & \\
\hline Trilema 3 & 2.27 & .71 & 2.41 & .62 & $-1.31(84)$ & .11 \\
\hline Trilema 5 & 2.69 & .57 & 2.77 & .45 & $-1.14(84)$ & .26 \\
\hline Trilema 7 & 2.79 & .55 & 2.85 & .43 & $-1.16(84)$ & .25 \\
\hline Trilema 11 & 2.20 & .72 & 2.26 & .68 & -.66 (84) & .51 \\
\hline Trilema 14 & 1.52 & .66 & 1.64 & .71 & $-2.00(84)$ & $.049 *$ \\
\hline Trilema 19 & 2.47 & .83 & 2.58 & .68 & $-2.04(84)$ & $.045^{*}$ \\
\hline Trilema 20 & 2.36 & .85 & 2.43 & .81 & $-1.09(84)$ & .28 \\
\hline \multicolumn{7}{|l|}{ Reconocimiento } \\
\hline & \multicolumn{2}{|c|}{ Pre } & \multicolumn{2}{|c|}{ Post } & & \\
\hline & $M$ & $D T$ & $M$ & $D T$ & $t(g l)$ & Sig. \\
\hline Trilema 1 & 2.24 & .84 & 2.53 & .62 & $-3.43(84)$ & $.001 *$ \\
\hline Trilema 2 & 1.86 & .77 & 1.99 & .70 & $-1.69(84)$ & $.005 *$ \\
\hline Trilema 6 & 2.85 & .40 & 2.89 & .31 & $-.90(84)$ & .37 \\
\hline Trilema 13 & 2.49 & .80 & 2.54 & .71 & $-.75(84)$ & .45 \\
\hline Trilema 16 & 1.99 & .49 & 2.08 & .52 & $-1.57(84)$ & $.048^{*}$ \\
\hline Trilema 17 & 2.68 & .52 & 2.69 & .53 & $-.33(84)$ & .74 \\
\hline Trilema 18 & 2.24 & .87 & 2.69 & .52 & $-4.42(84)$ & $.000^{*}$ \\
\hline \multicolumn{7}{|l|}{ Participación } \\
\hline & \multicolumn{2}{|c|}{ Pre } & \multicolumn{2}{|c|}{ Post } & & \\
\hline & $M$ & $D T$ & $M$ & $D T$ & $t(g l)$ & Sig. \\
\hline Trilema 4 & 2.65 & .65 & 2.85 & .43 & $-3.04(84)$ & $.003 *$ \\
\hline Trilema 8 & 2.35 & .65 & 2.89 & .49 & $-3.29(84)$ & $.002 *$ \\
\hline Trilema 9 & 2.12 & .40 & 2.35 & .58 & $-3.13(84)$ & $.003 *$ \\
\hline Trilema 10 & 1.92 & .79 & 2.26 & .62 & $-4.11(84)$ & $.000^{*}$ \\
\hline Trilema 12 & 1.91 & .85 & 2.27 & .76 & $-3.83(84)$ & $.000 *$ \\
\hline Trilema 15 & 2.27 & .90 & 2.50 & .67 & $-2.41(84)$ & $.018^{*}$ \\
\hline Trilema 21 & 2.47 & .78 & 2.55 & .67 & $-1.14(84)$ & .26 \\
\hline
\end{tabular}

Fuente: Elaboración propia a partir de los resultados de la investigación.

En relación a los resultados pretest-postest en función del sexo del alumnado, como se observa en la Tabla 3, en los alumnos se hallaron cambios significativos en las dimensiones Reconocimiento y Participación, no siendo así para la dimensión Redistribución. De la misma 
manera, las alumnas mostraron cambios pretest-postest en las dimensiones Reconocimiento y Participación.

Tabla 3. Puntuaciones promedio y diferencias pre-post programa según el sexo.

\begin{tabular}{|c|c|c|c|c|c|c|c|c|c|c|}
\hline & & $\begin{array}{c}\text { Redist. } \\
\text { Pre } \\
\end{array}$ & $\begin{array}{c}\text { Redist. } \\
\text { Post }\end{array}$ & Sig. & $\begin{array}{c}\text { Recon. } \\
\text { Pre }\end{array}$ & $\begin{array}{c}\text { Recon. } \\
\text { Post }\end{array}$ & Sig. & $\begin{array}{c}\text { Partic. } \\
\text { Pre } \\
\end{array}$ & $\begin{array}{c}\text { Partic. } \\
\text { Post }\end{array}$ & Sig. \\
\hline \multirow{2}{*}{ Alumnos } & $M$ & 16.26 & 16.84 & $p=.59$ & 16.25 & 17.17 & $p=.041$ & 15.46 & 17.45 & $p<.001$ \\
\hline & $D T$ & 2.26 & 2.18 & & 1.75 & 1.82 & & 2.38 & 1.90 & \\
\hline \multirow{2}{*}{ Alumnas } & $M$ & 16.50 & 17.07 & $p=.19$ & 16.65 & 17.71 & $p=.039$ & 16.07 & 17.25 & $p<.001$ \\
\hline & $D T$ & 2.08 & 1.84 & & 1.80 & 1.51 & & 1.80 & 1.89 & \\
\hline
\end{tabular}

Fuente: Elaboración propia a partir de los resultados de la investigación.

En este sentido, a nivel intragrupal los alumnos mostraron diferencias significativas pretest-postest en la dimensión Reconocimiento $\left(t_{(84)}=2.30 ; p=.041\right)$ y en la dimensión Participación $\left(t_{(84)}=4.98 ; p<.001\right)$. Por su parte, las alumnas también mostraron diferencias pretestpostest estadísticamente significativas en las dimensiones Reconocimiento $\left(t_{(84)}=4.03 ; p=.039\right)$ y Participación $\left(t_{(84)}=3.96 ; p<.001\right)$. En la dimensión Redistribución aunque también se hallaron cambios, las diferencias pretest-postest no fueron significativas ni en los alumnos $\left(t_{(84)}=.76 ; p=.59\right)$, ni en las alumnas $\left(t_{(84)}=.97 ; p=.19\right)$. A nivel intergrupal no se encontraron hallazgos significativos, siendo las alumnas quienes mostraron representaciones medias más prosociales para las dimensiones Redistribución y Reconocimiento, y los alumnos para la dimensión Participación.

Por último, en base a los resultados pretest-postest en función del centro educativo del alumnado, como se observa en la Tabla 4, se hallaron diferencias significativas en las dimensiones Reconocimiento y Participación, no siendo así para la dimensión Redistribución.

Tabla 4. Puntuaciones medias y diferencias pre-post programa según el tipo de centro.

\begin{tabular}{|c|c|c|c|c|c|c|c|c|c|c|}
\hline & & $\begin{array}{c}\text { Redist. } \\
\text { Pre }\end{array}$ & $\begin{array}{c}\text { Redist. } \\
\text { Post }\end{array}$ & Sig. & $\begin{array}{c}\text { Recon. } \\
\text { Pre }\end{array}$ & $\begin{array}{c}\text { Recon. } \\
\text { Post }\end{array}$ & Sig. & $\begin{array}{c}\text { Partic. } \\
\text { Pre }\end{array}$ & $\begin{array}{c}\text { Partic. } \\
\text { Post }\end{array}$ & Sig. \\
\hline \multirow{2}{*}{ Privado } & $M$ & 15.65 & 15.95 & $p=.98$ & 16.86 & 17.72 & $p=.036$ & 15.36 & 17.77 & $p<.001$ \\
\hline & DT & 2.03 & 1.80 & & 1.67 & 1.45 & & 2.15 & 1,84 & \\
\hline \multirow{2}{*}{ Público } & $M$ & 16.62 & 17.33 & $p=.58$ & 16.19 & 17.23 & $p=.029$ & 15.82 & 17.21 & $p<.001$ \\
\hline & DT & 2.19 & 2.02 & & 1.79 & 1.81 & & 2.21 & 1.90 & \\
\hline
\end{tabular}

Fuente: Elaboración propia a partir de los resultados de la investigación. 
A nivel intragrupal el alumnado perteneciente al centro de educación privada mostró representaciones significativamente más prosociales tras participar en el programa de intervención en la dimensión Reconocimiento $\left(t_{(84)}=2.18 ; p=.36\right)$ y en la dimensión Participación $\left(t_{(84)}=4.45 ; p<.01\right)$. Por su parte, el alumnado procedente de la educación pública también mostró representaciones post-intervención significativamente más prosociales en las dimensiones Reconocimiento $\left(t_{(84)}=3.67 ; p=.029\right)$ y Participación $\left(t_{(84)}=4.89 ; p<.001\right)$. En la dimensión Redistribución aunque también se hallaron mejoras pre-post intervención, estas no fueron significativas ni en el centro privado $\left(t_{(84)}=.12 ; p=.98\right)$, ni en los centros públicos $\left(t_{(84)}=.78\right.$; $p=.58)$. A nivel intergrupal no se encontraron diferencias significativas entre las puntuaciones pretest-postest en función del tipo de centro educativo. No obstante, fue el alumnado de los centros públicos quien mostró puntuaciones mayores pretest y postest en la dimensión Reconocimiento, así como mayores puntuaciones postest en la dimensión Participación. En el caso del alumnado perteneciente a la educación privada, estos mostraron puntuaciones pretest y postest más elevadas en la dimensión Redistribución, así como mayores puntuaciones pretest en la dimensión Participación.

\section{Discusión y Conclusiones}

El presente estudio contribuye a la comprensión de los espacios y profesionales educativos como posibles agentes para la transformación y la mejora del alumnado, en términos de prosocialidad. En este sentido, la implementación preliminar del programa socioeducativo diseñado se ha mostrado eficaz para mejorar la prosocialidad en las representaciones de Justicia Social del alumnado de $6^{\circ}$ curso de educación primaria. Desde la perspectiva del alumnado, el profesorado modifica sus habilidades al abordarlas cuando realizan actividades académicas, tanto en términos de sus habilidades como en sus procedimientos de trabajo y participación en las clases (Lobos, Bustos y Díaz, 2019). Sin embargo, también es relevante contar con un entorno educativo orientado hacia la búsqueda explícita de la Justicia Social. Así, el alumnado no solo toma el espacio educativo como un lugar en donde adquirir aprendizajes instrumentales sino también como un espacio para desarrollarse como personas, responsables de si mismas y del mundo en que viven (Perines e Hidalgo, 2018). En este sentido, el programa implementado tiene una clara orientación hacia el establecimiento de modelos de convivencia prosociales en el espacio educativo, mediante actividades y talleres (e. g. mural grupal de convivencia o técnica de role-play) que buscan proporcionar referentes y estrategias estables para la resolución y prevención de situaciones injustas. 
Tras la implementación preliminar del programa diseñado fueron encontrados cambios en los indices de prosocialidad hacia las tres dimensiones de Justicia Social evaluadas, siendo significativos los cambios en las dimensiones reconocimiento y participación. El alumnado mejoró particularmente su prosocialidad hacia la dimension participación, pudiendo ello relacionarse con la transversalidad que posee esta dimensión para la consecución de la redistribución y el reconocimiento (Sainz, 2017). En los tres módulos de intervención fue planteada la participación como clave para que el alumnado pueda lograr solucionar de manera justa diversos trilemas teórico-prácticas. En este sentido, el programa se muestra en consonancia con el "principio de participación paritaria" (Fraser, 2008) según el cual la justicia social no se consigue por la mera transmisión de conocimientos en torno a la redistribución y al reconocimiento sino que precisa de una participación efectiva de todos las partes implicadas. Es importante destacar que al tratarse de una experiencia innovadora, no existen antecedentes de estudios que hayan trabajado la prosocialidad evaluada mediante trilemas basados en la justiciar social. No obstante, si existen antecedentes de programas de intervención de promoción de la prosocialidad en educación primaria, aunque basados directamente en variables como la empatía y la inteligencia emocional. Así, diversos estudios se muestran coherentes con la importancia de la dimensión participación como una de las formas más efectivas de alentar la prosocialidad (Spinrad y Gal, 2018).

Atendiendo al sexo del alumnado, los hallazgos son coherentes con los mostrados por estudios descriptivos en educación primaria y niveles superiores (Sainz y Jacott, 2020; Juanes, Jacott y Maldonado, 2017). De cualquier forma, una vez implementado el programa preliminar, tanto las alumnas como los alumnos mejoraron su nivel de prosocialidad, resolviendo de media una mayor cantidad de trilemas de manera justa. Estos datos reflejan que con intervenciones socioeducativas es posible promover la prosocialidad mediante principios basados en la Justicia Social entre el alumnado (y futuros/as ciudadanos/as) independientemente de su sexo. Así, este tipo de intervenciones escolares suponen una medida educativa que puede conseguir mejorar la resolución de conflictos, la prevención de situaciones de acoso y un mayor desarrollo personal (Pérez-Albarracín y Fernández-Baena, 2019). En el futuro, se sugiere continuar estudiando en profundidad las diferencias entre alumnas y alumnos en las tres dimensiones analizadas, así como otras variables psicoeducativas relacionadas. 
En base al tipo de centro educativo, es destacable que en el centro educativo privado el alumnado muestra una menor prosocialidad hacia los trilemas relacionados con la dimensión redistribución. En este sentido, es relevante destacar que en Madrid (España) el 77,6\% del alumnado extranjero está escolarizado en la escuela pública y el 22,9\% en la privada (Goenechea, 2016), por lo cual en los centros privados el perfil del alumnado podría ser más homogéneo que en los centros públicos. Con ello, en los centros públicos existe una mayor diversidad socioeconómica y cultural, y esto podría hacer que algunas cuestiones relacionadas con la justicia social, especialmente en la dimensión redistribución, estén más presentes en las aulas de la educación pública que las de la educación privada. No obstante, en las dimensiones reconocimiento y participación los niveles de prososocialidad iniciales y las mejoras del alumnado han sido similares, independientemente del tipo de centro educativo. De cara a futuros estudios se sugiere ampliar la implementación a la educación concertada, así como a una mayor diversidad de centros privados y públicos. En este sentido, para futuros estudios se sugiere agregar al análisis diversas variables familiares que pudieran mostrar diferencias en base diversos patrones educativos ya que, siguiendo a Garaigordobil (2014), la conducta prosocial de los niños puede verse positivamente influenciada en función del apego, altruismo y modelo comportamental de los padres.

En cuanto a la metodología de estudio, para que el programa sea más efectivo y tenga un mayor impacto se considera pertinente prolongar su implementación e incluso implantarlo de forma estable en los centros junto a otras actuaciones psicoeducativas. Además, supondría un avance metodológico ampliar la evaluación del programa una vez se implemente de forma estable en los centros. En este sentido, siguiendo a Pérez Juste (2006); Pérez-González (2008), se sugiere seguir una evaluación en tres momentos concretos: una evaluación inicial que evalúe el programa en base a su adecuación a los destinatarios y al contexto; una evaluación procesual o continua coincidente con el período de implementación del programa; y una evaluación final o sumativa relacionada con los resultados o logros del programa, incluyendo la valoración acerca de los mismos.

En el caso del presente estudio, se llevó a cabo una evaluación con diseño pretestpostest, que se complementará en futuros estudios con una evaluación de implementación. Siguiendo a Bono Cabré (2012), en futuras investigaciones asociadas a la implementación del programa se agregarán grupos de participantes control para aumentar la validez interna de los resultados. Una limitación del presente estudio ha sido la mejora desigual en la prosocialidad 
hacia cada dimensión de la Justicia Social. En este sentido, cabe reflexionar acerca de la efectividad del enfoque asumido en el que la participación asumió un mayor peso como acción para la consecución de la prosocialidad. De cara a futuras experiencias, cabría explicitar con mayor profundidad al alumnado la inclusión de la redistribución y el reconocimiento como elementos necesarios para la resolución de injusticias sociales. De la misma manera, en futuros estudios se complementará la evaluación mediante la metodología cualitativa, ya que según Morgade, Poveda y González-Patiño (2014) “esta intenta además entender cómo se enfrentan las/os niñas/os a sus entornos, cómo se adaptan e integran los recursos que se les ofrecen y cómo adaptan su forma de vida en función de aquello a lo que acceden” (p. 764).

Durante años, la crisis socioeconómica ha contribuido a crear sociedades cada vez más desiguales e injustas (Sotomayor, Merklen y García-Domingo, 2017). En este sentido, uno de los principales pilares para mejorar y transformar esta situación debería incluir una Educación para la Justicia Social (Murillo y Hernández-Castilla, 2011). De esta forma, en el futuro los y las estudiantes podrán transformar la sociedad y revertir las situaciones de injusticia individuales y colectivas. Así, aplicar los contenidos del modelo tridimensional de Justicia Social (Fraser, 2008) se sitúa como una herramienta para comprender el origen de estas opresiones y participar desde la educación para la transformación social. Todo ello comprende diversas formas de dar sentido al papel que las prácticas socioeducativas tendrán en el desarrollo del alumnado, viéndose involucrados de forma sistémica diversos espacios en este proceso: la escuela, la ciudad, el hogar, entre otros (Morgade, Poveda y González-Patiño, 2014). Así, se considera necesaria la implicación de las instituciones educativas ya que, por ejemplo, en términos de justicia económica o redistribución, un sistema justo no puede en ningún grado victimizar (o premiar) a las personas por la suerte o desgracia en que ha nacido (y, por tanto, no ha elegido), al contrario, debe tender activamente a contrarrestarlo (Bolivar, 2012). Por ello, se concluye que es fundamental continuar profundizando en un debate fundamentado y multidisciplinar, a partir del cual se puedan tomar en consideración propuestas educativas aplicadas para promover la prosocialidad, estudiando diversos factores y variables psicosociales y socioeducativas. 


\section{Referencias}

Balsells, M. À., Peláez, N. F., Gomà, M. M., Inchaurrondo, A. M., \& Hoz, V. V. (2010). Innovación socioeducativa para el apoyo de adolescentes en situación de acogimiento familiar. Educar, 45, 133-148. Recuperado de: https://www.raco.cat/index.php/Educar/article/view/214612

Bell, L. A. (1997). Theoretical foundations for social justice education. En M. Adams, L. A. Bell y P. Griffin (Eds.), Teaching for diversity and social justice: A sourcebook. Nueva York: Routledge.

Bolívar, A. (2012). Justicia social y equidad escolar, una revisión actual. Revista Internacional de Educación para la Justicia Social, 1 (1), 9-45. Recuperado de: http://hdl.handle.net/10486/9162

Bono Cabré, R. (2012). Diseños cuasi-experimentales y longitudinales. Barcelona: Universidad de Barcelona.

Cabrera, C. y Davyt, A. (2015). Aprendizaje cooperativo en estudiantes de ciencias exactas y naturales: el "Programa Compromiso Educativo". Revista Iberoamericana de Educación, 67(1), 203-216. Recuperado de: https://rieoei.org/historico/expe/6739Cabrera.pdf

Cerchiaro-Ceballos, E., Barras-Rodríguez, R., y Vargas-Romero, H. (2019). Juegos cooperativos y razonamiento prosocial en niños: efectos de un programa de intervención. Duazary, 16(3), 4053. Doi: https://doi.org/10.21676/2389783X.2967

Cole, M. (2000). Education, equality and human rights: Issues of gender, 'race,' sexuality, special needs and social class. Londres: Routledge.

Di Lorenzo, M. (2012). Nuevas formas de violencia entre pares: del bullying al cyberbullying. Revista Médica del Uruguay, 28(1), 48-53. Recuperado de: http://www.scielo.edu.uy/scielo.php?script=sci_arttext\&pid=S168803902012000100007\&lng=es\&tlng=

Duque, M. C. C. (2017). Aproximaciones epistemológicas y conceptuales de la conducta prosocial. Revista del Instituto de Estudios Superiores en Educación, 27, 1-21. Doi: 10.14482/zp.27.10978

Dworkin, R. (1981). What is Equality? Equality of Resources. Philosophy \& Public Affairs, 10 (4), 283-345. Recuperado de: www.jstor.org/stable/2264894

Eisenbraun, K. (2007). Violence in School: Prevalence, Prediction, and Prevention. Aggression and Violent Behavior, 12, 459-469. Doi: https://doi.org/10.1016/j.avb.2006.09.008

Fraser, N. (1997). Justice interuptus. Critical reflextions on the "Postsocialist" Condition. Nueva York: Routledge.

Fraser. N. (2008). Scales of Justice. New York: Columbia University Press.

Garaigordobil, M. y Oñederra, J.A. (2008). Estudios epidemiológicos sobre la incidencia del acoso escolar e implicaciones educativas. Información Psicológica, 94, 14-35. Recuperado de: 
http://www.informaciopsicologica.info/OJSmottif/index.php/leonardo/article/view/236

Garaigordobil, M. (2003). Intervención psicológica para desarrollar la personalidad infantil. Juego, conducta prosocial y creatividad. Madrid: Pirámide.

Garaigordobil, M. (2014). Conducta prosocial: el papel de la cultura, la familia, la escuela y la personalidad. Revista Mexicana de investigación en Psicología, 6(2), 146-157. Recuperado de: https://www.medigraphic.com/cgi-bin/new/resumen.cgi?IDARTICULO=70550

García-Lastra, M. (2013). Educar en la sociedad contemporánea: Hacia un nuevo escenario educativo.

Convergencia, 20(62), 199-220. Recuperado de: https://convergencia.uaemex.mx/article/view/1042

Goenechea, C. (2016). La escuela pública de la España multicultural. Revista interuniversitaria de formación del profesorado, 85, 111-119. Doi: https://doi.org/10.9685/i2863

Gómez, E. N. y Alatorre, F. J. (2014). La intervención socioeducativa: Cuando se juega en la cancha del otro. Sinéctica, 43, 01-17. Recuperado de: http://www.scielo.org.mx/scielo.php?script=sci_arttext\&pid=S1665109X2014000200002\&lng=es\&tlng=es

Irvine, J. (2003). Educating teachers for diversity: Seeing with a cultural eye. Nueva York: Teachers College Press.

Jacott, L. y Maldonado, A. (2012). La educación para la ciudadanía: Nuevos retos y perspectivas desde el enfoque de la justicia social. Revista Internacional de Educación para la Justicia Social, 2(1), 5-13. Recuperado de: http://hdl.handle.net/10486/13532

Jacott, L., Maldonado, A., Sainz, V., Juanes, A., García-Vélez, T. y Seguro, V. (2014). Representations of social justice amongst Spanish teachers and students. En P. Cunningham y N. Fretwell (Eds.), Innovative Practice and Research Trends in Identity, Citizenship and Education. Londres: CiCe.

Jiménez, F., Lalueza, J. L., y Fardella, C. (2017). Aprendizajes, inclusión y justicia social en entornos educativos multiculturales. Revista electrónica de investigación educativa, 19(3), 10-23. Doi: https://dx.doi.org/10.24320/redie.2017.19.3.830

Jornet-Meliá, J.M. Sancho-Álvarez, C. y Bakieva, M. (2015). Diseño y validación del constructo teórico de Justicia Social percibida por el alumnado de centros escolares. Revista Internacional de Educación para la Justicia Social, 4(2), 111-126.

Juanes, A. (2018). Representaciones de justicia social en estudiantes de Educación Primaria. Estudio cuantitativo y cualitativo comparado entre España y Argentina (Tesis Doctoral). Universidad Autónoma de Madrid, España. Recuperado de: http://hdl.handle.net/10486/682707

Juanes, A., Jacott, L. y Maldonado, A. (2017). Representaciones de justicia social en profesores en formación. Revista Internacional de Didáctica y Organización Educativa, 3(2), 26-45. Doi: http://dx.doi.org/10.15366/riejs2015.4.2 
Juanes, A., Sainz, V. Garcia, T., Maldonado, A., y Jacott, L. (2016). Social justice: A qualitative and quantitative study of representations of social justice in children of primary education. SHS Web of Conferences 26, 1-5. Doi: https://doi.org/10.1051/shsconf/20162601054

Lee, C.C. e Hipólito-Delgado, C. (2007). Counselors as agents of social justice. In C.C. Lee (Ed.). Counseling for Social Justice, 13-38. Alexandria: American Counseling Association.

Lozano, J. S. H., \& Lechuga, B. M. (2019). Programa psicoeducativo para desarrollar la conducta prosocial en niños de edad escolar. In IX Congreso Estudiantil y IV Congreso internacional de investigación en Psicología. México: FES Zaragoza.

Martínez, E. (2002). “Aporofobia”. En J. Conill (presidente). Glosario para una Sociedad Intercultural. Bancaja, Valencia.

Mateu-Martínez, O., Piqueras, J.A., Rivera-Riquelme, Espada, J.O. y Orgilés, M. (2014). Aceptación /rechazo social infantil: relación con problemas emociones e inteligencia emocional. Avances en Psicología, 22(2), 205-213. Doi: https://doi.org/10.33539/avpsicol.2014.v22n2.190

McAuliffe, M. D., Hubbard, J. A., \& Romano, L. J. (2009). The role of teacher cognition and behavior in children's peer relations. Journal of abnormal child psychology, 37(5), 665-677. Doi: https://doi.org/10.1007/s10802-009-9305-5

Miller, S., Williamson, P., George, M., King, J., Charest, B., Bieler, D., \& Bolf Beliveau, D. (2011). Extending the conversation: Applying the CEE position statement 'beliefs about social justice in English education' to classroom praxis. English Education, 44 (1), 63-80. Recuperado de: https://www.jstor.org/stable/23238723

Monjas, M. I., Martín-Antón, L., García-Bacete, F.J. y Sanchiz, M. L. (2014). Rechazo y victimización al alumnado con necesidad de apoyo educativo en primero de primaria. Anales de Psicología, 30 (2). Doi: https://dx.doi.org/10.6018/analesps.30.2.158211

Morgade, M. S., Poveda, D., \& González-Patiño, J. (2014). Del hogar a la ciudad como camino de ida y vuelta en el desarrollo de la identidad: El caso de las rutinas de la infancia urbana de clase media/alta en Madrid. Educação e Sociedade, 35 (128), 761-780. Recuperado de: http://hdl.handle.net/10486/667078

Murillo, F.J. y Hernández Castilla, R. (2011). Hacia un concepto de justicia social. Revista Iberoamericana sobre Calidad, Eficacia y Cambio en Educación, 9 (4), 7-23. Recuperado de: http://www.rinace.net/reice/numeros/arts/vol9num2/art1.pdf

Murillo, F.J. y Hernández-Castilla, R. (2014). Liderando Escuelas Justas para la Justicia Social. Revista Internacional de Educación para la Justicia Social, 3(2), 13-32. Recuperado de: https://revistas.uam.es/riejs/article/view/337

Murillo, F.J. y Krichesky, G.J. (2012). El proceso del cambio escolar. Una guía para impulsar y sostener la mejora en las escuelas. Revista Iberoamericana sobre Calidad, Eficacia y Cambio en $\begin{array}{llll}\text { Educación, } & 10 & \text { (10), } & \text { Recuperado de: }\end{array}$ http://www.rinace.net/reice/numeros/arts/vol10num1/art2.pdf 
Nocito Muñoz, G. (2017). Investigaciones sobre el acoso escolar en España: implicacines psicoeducativas. REOP-Revista Española de Orientación y Psicopedagogía, 28(1), 104-118. Doi: doi.org/10.5944/reop.vol.28.num.1.2017.19361

Olweus D. (1994) Bullying at schools: basic facts and effects of a school-based intervention program. Child Psychol Psychiatry, 35(7), 1171-1190. Doi: 10.1111/j.1469-7610.1994.tb01229.x

Peña, K. L., Bustos, C., \& Díaz, A. (2019). Impact of teacher training on academic self-concept and educational outcomes. Electronic Journal of Research in Education Psychology, 17(49), 519540. Doi: http://dx.doi.org/10.25115/ejrep.v17i49.2219

Pérez-Albarracín, A. y Fernández-Baena, J. (2019). Beyond conflict resolution: socio-emotional learning in student mediators. Electronic Journal of Research in Educational Psychology, 17(2), 335-358. Doi: http://dx.doi.org/10.25115/ejrep.v17i48.2223

Pérez-González, J.C. (2008). A proposal for evaluating socio-emotional education programs. Electronic Journal of Research in Educational Psychology, 6 (2), 522-546. Doi: http://dx.doi.org/10.25115/ejrep.v6i15.1285

Perines, H. e Hidalgo, N. (2018). "La escuela confía en que los estudiantes podemos cambiar el mundo": un estudio de las escuelas que trabajan para la justicia social. Revista Colombiana de Educación, 75, 19-38. Doi: https://doi.org/10.17227/rce.num75-8099

Rajmil, L, Alonso, J. Analitis, F., Detmar, S., Erhart, M., Klein, M., Ravens-Sieberer, U., Herdman, M., y Berra, S. (2009). Víctimas de acoso: factores asociados en niños y adolescentes de 8 -18 años de edad en 11 países europeos. Pedriatrics, 67 (2), 111-118. Recuperado de: https://www.elsevier.es/es-revista-pediatrics-10-pdf-13134910

Rappaport, J. (1977). Community psychology: Values, research and action. Nueva York: Rinehart and Winston.

Rawls, J. A. (1971). A theory of justice. Cambridge: Harvard University Press.

Sainz, V. (2017). Representaciones de la justicia social en profesores y estudiantes de Educación Secundaria (Tesis Doctoral, Universidad Autónoma de Madrid). Recuperado de: http://hdl.handle.net/10486/680617

Sainz, V. y Jacott, L. (2020). What Do Secondary Education Students Understand About Social Justice? Students Representations of Social Justice in Different Regions of Spain. Interchange, 51, 1-22. Doi: https://doi.org/10.1007/s10780-020-09389-y

Sampieri, H., Collado, F., \& Lucio, B. (2014). Metodología de la investigación. México D.F.: McGraw-Hill.

Sánchez, C. (2009). Nivel de implicación en bullying entre escolares de educación primaria. Relación con el estatus sociométrico y la percepción del clima social familiar y escolar (Tesis doctoral. Murcia, Universidad de Murcia). Recuperado de: https://www.tesisenred.net/handle/10803/11028\#page=1

Sen, A. (2009). The idea of Justice. Nueva York: Penguin Press. Madrid: Taurus. 
Sensoy, O. y DiAngelo, R. (2017). Is everyone really equal?: An introduction to key concepts in social justice education. New York: Teachers College Press.

Simón, C., Barrios, Á., Gutiérrez, H., y Muñoz, Y. (2019). Equidad, Educación Inclusiva y Educación para la Justicia Social. ¿Llevan Todos los Caminos a la Misma Meta?. Revista Internacional de Educación para la Justicia Social, 8(2), 17-32. Doi: https://doi.org/10.15366/riejs2019.8.2.001

Smith, E. 2018. Key issues in education and social justice. London: Sage.

Sotomayor, E., Merklen, D. y García-Domingo, M. (2017). Transformaciones sociales en la Europa de la crisis: la gestación de la desigualdad social. Index de Enfermería, 26(3), 185-189. Recuperado de: http://www.index-f.com/index-enfermeria/v26n3/2635.php

Spinrad, T. L., \& Gal, D. E. (2018). Fostering prosocial behavior and empathy in young children. Current Opinion in Psychology, 20, 40-44. Doi: 10.1016/j.copsyc.2017.08.004

Wartenweiler, T. (2018). Serious play in education for social justice-An exploratory study. Journal of New Approaches in Educational Research, 7(1), 61-69. Doi: 10.7821/naer.2018.1.260

Yáñez, S. V. (1996). Preferencias perceptuales de estilo de aprendizaje en cuatro escuelas primarias. Revista mexicana de investigación educativa, 1(2), 283-313. Recuperado de: https://www.redalyc.org/articulo.oa?id=140/14000203

Zimmerman, P. (2000). Empowerment Theory: Psychological, Organizational and Community Levels of Analysis. En J. Rappaport y E. Seidman (Eds.), Handbook of Community Psychology. Nueva York: Kluwer Academic Plenum.

Recibido: $30-03-2020$

Aceptado: $28-05-2020$ 